J. Lake Sci. (湖泊科学), $2007, \mathbf{1 9}(4): 434-444$

http://www. jlakes.org. E-mail: jlakes@ niglas. ac.cn

(c) 2007 by Journal of Lake Sciences

\title{
鄱阳湖鱼类资源及渔业利用
}

张堂林, 李钟杰

(中国科学院水生生物研究所,武汉 430072)

摘 要: 1997 年冬至 2000 年春,对我国最大淡水湖鄱阳湖的鱼类与渔业利用状况进行了调查. 在 1955 年至 2000 年期 间, 鄱阳湖累计记录鱼类 136 种, 隶属于 25 科 78 属; 其中鲤科鱼类最多,有 71 种,占总种数的 $52.2 \%$,其次是魭科, 12 种, 占 $8.8 \%$. 本次调查期间共记录鱼类 101 种,新记录鱼类 6 种,即亮银鮈、洞庭小鯂鮈、光唇蛇鮈、短须鱊、方氏鳑鲏和粘皮 鲻鰕虎鱼. 还分析了该湖鱼类区系组成的变化、主要渔具的渔获物组成特征以及渔产量动态, 提出了未来湖泊渔业可持 续发展的建议.

关键词: 鱼类区系;渔业资源;渔业管理;鄱阳湖

\section{Fish resources and fishery utilization of Lake Poyang}

ZHANG Tanglin \& LI Zhongjie

(Institute of Hydrobiology, The Chinese Academy of Sciences, Wuhan 430072, P. R. China)

\begin{abstract}
An investigation on fish resources and fishery utilization of Lake Poyang, the largest freshwater lake in China, was carried out from winter 1997 to spring 2000. During the period of $1955-2000$, the accumulated number of 136 species, belonging to 25 families and 78 genera, was recorded in Lake Poyang. The largest of families was the Cyprinidae ( 71 species), contributing $52.2 \%$ to the total; the second was the Bagridae ( 12 species), accounting for $8.8 \%$. During this investigation, 101 species were recorded, and 6 new records were found, which were Squalidus nitens (Günther), Microphysogobio tungtingensis (Nichols), Saurogobio gymnocheilus Lo, Yao et Chen, Acheilognathus barbatulus Günther, Rhodeus fangi (Miao), Mugilogobius myxodermus (Herre). Moreover, fish composition in catch from several important fishing gears and the dynamic change in fish yield were analyzed, and some suggestions on the sustainable fisheries development were proposed.
\end{abstract}

Keywords : Ichthyologic fauna; fishery resources; fishery management; Lake Poyang

鄱阳湖是中国最大的淡水湖泊,也是目前直接通江的重要湖泊. 该湖汇纳赣江、抚河、信江、饶河、修水 五条河流之水, 经调蓄后, 通过湖口注人长江. 水位涨落直接受到长江与 “五河” 水情的控制, 水位落差大 ( 7 $\mathrm{m}$ 以上), 是一个典型的过水性吞吐型湖泊.

鄱阳湖由于江、湖之间物质和能量的频繁交换,加上静水、流水生境的互补作用,孕育出相当复杂的淡 水生物群落,鱼类资源丰富. 该湖既是江湖洄游性鱼类重要的摄食和育肥场所,也是某些过河口洄游性鱼 类的繁殖通道或繁殖场, 对长江鱼类种质资源保护及种群的维持具有重大意义.

虽然鄱阳湖鱼类已作过一些工作 ${ }^{[1-5]}$,但是仍然有必要再次调查整理. 主要原因有二: 一是近年来某 些科下阶元的名称或所属关系有所变更,某些鱼类学名被重新订正, 以致某些种名失效或存在同物异 名 ${ }^{[6-10]}$; 再是水利建设、围湖造田、江河污染及渔业活动对鄱阳湖的影响日渐明显, 进而影响到鱼类资源, 而原有的调查工作大都是在 20 世纪 80 年代以前开展的. 鉴于此,1997 年冬季至 2000 年春季,我们先后对 鄱阳湖鱼类与渔业进行了调查, 内容包括鱼类区系、鱼类生态类型、渔具渔法、渔获物组成和渔业管理方面,

* 中国科学院重大项目 (KZ951-A1-102,KZ951-B1-104) 资助. 2006-07-13 收稿; 2006-11-06 收修改稿. 张堂林, 男,1966 年生,博士, 副研究员; E-mail: tlzhang@ihb. ac. cn. 
旨在积累基础资料,为鄱阳湖鱼类资源的保护与可持续利用提供科学依据.

\section{1 材料与方法}

本次鱼类调查的范围比较广, 涉及到沿湖 7 个县市 (湖口、都昌、波阳、新建、永修、进贤和南昌) 所在的 湖区,所查的鱼类样本主要来自网䈤、钓钩、电捕、刺网、蹦网 ( pop-net) 和虾笼等多种渔具. 鱼类分类地位、 中文名和学名的确定是依据最新的鱼类分类学资料, 主要参照 《中国淡水鱼类检索》 ${ }^{[6]}$ 《中国动物志一 硬骨鱼纲 鲤形目》 ${ }^{[7,8]}$ 、《中国动物志一一硬骨鱼纲 鲇形目》 ${ }^{[9]}$ 和《拉汉世界鱼类名典》 ${ }^{[10]}$.

由于鄱阳湖面积大, 捕捞作业分散, 若要分析整个湖区的渔获物组成, 则非常困难, 因此, 只对主要渔具 的渔获物组成特征进行了调查. 在每次调查时, 若某种渔具的渔获物少,则把所有鱼类进行分类统计; 若渔 获物多, 则随机抽样. 虽然这种调查所得的数据难以反映鄱阳湖渔获物组成的全貌, 但至少可以说明哪些 鱼类资源比较丰富, 是鄱阳湖渔业的主要基础.

由于作业渔船分散,渔货自产自销,难以准确统计全湖渔产量. 为了对鄱阳湖渔产量有个粗略的估算, 根据湖区 11 个县市的渔业统计资料,将各县、市天然水域的捕捞量之和视为鄱阳湖渔产量, 且鄱阳湖面积 以 $2520 \mathrm{~km}^{2}$ 计 ${ }^{[5]}$. 根据江西省水产局和鄱阳湖管理局提供的相关资料,估算了 $1994-1997$ 年全湖的渔产 量. 为了分析鄱阳湖渔产量的变动特征, 还引用了 $1950-1993$ 年的渔产量数据 ${ }^{[5]}$.

\section{2 结果}

\section{1 鱼类区系组成及变化}

到目前为止,鄱阳湖累计记录鱼类 136 种,隶属于 25 科 78 属. 其中,鲤科鱼类最多,有 71 种, 占鱼类总 种数的 $52.2 \%$; 其次是魭科, 12 种,占 $8.8 \%$; 鲉科 8 种, 占 $5.9 \%$, 银鱼科和鮨科分别 5 种, 各占 $3.7 \%$, 其它

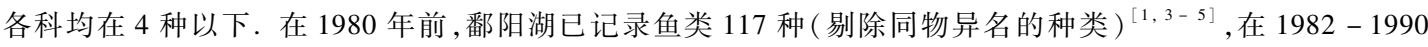
年, 记录鱼类 103 种 $^{[2]}$. 在本次调查期间记录的鱼类 101 种,其中新记录的鱼类有 6 种, 即亮银鮈 Squalidus nitens (Günther)、洞庭小鳔鮈 Microphysogobio tungtingensis (Nichols)、光唇蛇鮈 Saurogobio gymnocheilus Lo, Yao et Chen、短须鱊Acheilognathus barbatulus Günther、方氏鳑鲏 Rhodeus fangi（Miao）, 粘皮鲻鰕虎鱼 Mugilogobius myxodermus (Herre). 为了便于比较,特将以前报道过的和本次调查到的鱼类一并列入表 1 .

表 1 鄱阳湖鱼类种类名录

Tab. 1 Fish species recorded in Lake Poyang

鱼 类

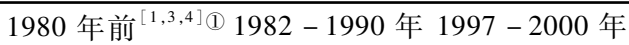

鲟科 Acipenseridae

1 中华鲟 Acipenser sinensis Gray

2 白鲟 Psephurus gladius (Martens)

鲱科 Engraulidae

3 鲋 Macrura reevesii (Richardson)

鯷科 Engraulidae ${ }^{[6,10]}$

4 鲚 Coilia ectenes Jordan et Seale

5 短领鲚 Coilia brachygnathus

(Kreyenberg et Pappenheim)

银鱼科 Salangidae ${ }^{[6,10]}$

6 太湖新银鱼 Neosalanx taihuensis Chen

7 乔氏新银鱼 Neosalanx jordani Wakiya et Takahasi

8 寡齿新银鱼 Neosalanx oligodonis Chen

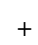

$+$

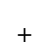

$+$

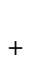

$+$

$+$ 
续表 1

9 大银鱼 Protosalanx hyalocranius ( Abbot)

10 短吻间银鱼 Hemisalanx brachyrostralis (Fang)

鳗鲟科 Anguillidae

11 鳗鲡 Anguilla japonica Temminck et Schlegel 胭脂鱼科 Catostomidae

12 胭脂鱼 Myxocyprinus asiaticus (Bleeker) 鲤科 Cyprinidae ${ }^{[7-8,10]}$

鿕亚科 Danioniae

13 宽鳍鱲 Zacco platypus ( Temminck et Schlegel)

14 马口鱼 Opsariichthys bidens Günther

雅罗鱼亚科 Leuciscinae

15 尖头鲴 Phoxinus oxycephalas (Sauvage et Dabry)

16 青鱼 Mylopharyngodon piceus (Richardson)

17 草鱼 Ctenopharyngodon idellus (Cuvier et Valenciennes)

18 赤眼鳟 Squaliobarbus curriculus (Richardson)

19 鳤Ochetobius elongatus (Kner)

20 鯮 Luciobrama macrocephalus (Lacepède)

21 鳡 Elopichthys bambusa (Richardson)

鲌亚科 Culterinae

22 飘鱼Pseudolaubuca sinensis Bleeker

23 寡鳞飘鱼 Pseudolaubuca engraulis (Nichols)

24 似鲚 Toxabramis swinhonis Günther

25 㛑 Hemiculter leucisculus (Basilewsky)

26 贝氏㛑 Hemiculter bleekeri Warpachowsky

27 红鯺原鲌 Culterichthys erythropterus Basilewsky

28 尧嘴鲌 Culter alburnus Basilewsky

29 蒙古鲌 Culter mongolicus (Basilewsky)

30 达氏鲌 Culter dabryi Bleeker

31 尖头鲌 Culter oxycephalus Bleeker

32 拟尖头鲌 Culter oxycephaloides Kreyenberg et Pappenheim

33 鳊 Parabramis pekinensis (Basilewsky)

34 鲂 Megalobrama skolkovii Dybowsky

35 团头鲂 M. amblycephala Yih

鲖亚科 Xenocyprinae

36 银鲖 Xenocypris argentea Günther

37 黄尾鲖 Xenocypris davidi Bleeker

38 细鳞鲖 Xenocypris microlepis Bleeker

39 似鳊 Pseudobrama simoni (Bleeker)

鲢亚科 Hypophthalmichthyinae

40 鲢 Hypophthalmichthys molitrix (Cuvier et Valenciennes)

41 鳙 Aristichthy nobilis (Richardson)

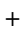


续表 1

鮈亚科 Gobioninae

42 唇鱼骨 Hemibarbus labeo Pallas

43 花䱉 Hemibarbus maculatus Bleeker

44 似刺鳊鮈 Paracanthobrama guichenoti Bleeker

45 麦穗鱼 Pseudorasbora parva (Temminck et Schlegel)

46 长麦穗鱼 Pseudorasbora elongata $\mathrm{Wu}$

47 华鳈 Sarcocheilichthys sinensis Bleeker

48 小鳈 Sarcocheilichthys parvus Nichols

49 江西鳈 Sarcocheilichthys kiangsiensis Nichols

50 黑鳍鳈 Sarcocheilichthys nigripinnis (Günther)

51 短须领须鮈 Gnathopogon imberbis Sauvage et Dabry

52 银鮈 Squalidus argentatus (Sauvage et Dabry)

53 亮银鮈 Squalidus nitens (Günther)

54 点纹银鮈 Squalidus wolterdstorffi (Regan)

55 铜鱼 Coreius heteroden (Bleeker)

56 北方铜鱼 Coreius septentrionalis (Nichols)

57 吻鮈 Rhinogobio typus Bleeker

58 圆筒吻鮈 Rhinogobio cylindricns Günther

59 棒花鱼 Abbottina rivularis (Basilewsky)

60 洞庭小鳔鮈 Microphysogobio tungtingensis (Nichols)

61 蛇鮈 Saurogobio dabryi Bleeker

62 长蛇鮈 Saurogobio dumerili Bleeker

63 光唇蛇鮈 Saurogobio gymnocheilus Lo, Yao et Chen 鱾鮀亚科 Gobiotinae

64 宜昌鱾鮀 Gobiobotia filifer (Garman)

鱊亚科 Acheilognathinae

65 无须鱊 Acheilognathus gracilis Nichols

66 大䱜鱊 Acheilognathus macropterus (Bleeker)

67 兴凯鱊Acheilognathus chankaensis Dybowsky

68 越南鱊Acheilognathus tonkinensis (Vaillant)

69 短须鱊 Acheilognathus barbatulus Günther

70 臭鳞鱊 Acheilognathus hyposelonotus (Bleeker)

71 巨口鱊 Acheilognathus tabira Jordan et Thompson

72 长身鱊 Acheilognathus elongatus ( Regan)

73 革条副鱊 Paracheilognathus himategus (Günther)

74 彩副鱊 Paracheilognathus imberbis (Günther)

75 高体鳑鲏 Rhodeus ocellatus (Kner)

76 彩石鳑鲏 Rhodeus lighti ( Wu)

77 方氏鳑鲏 Rhodeus fangi (Miao)

鲃亚科 Barbinae

78 光倒刺鲃 Spinibarbus hollandi Oshima

79 台湾光唇鱼 Acrossocheilus formosanus (Regan) 
续表 1

80 光唇鱼 Acrossocheilus fasciatus Steindachner

81 稀有白甲鱼 Onychostomas rara (Lin)

鲤亚科 Cyprininae

82 鲤 Cyprinus carpio Linnaeus

83 鲫 Carassius auratus (Linnaeus)

鱾科 Cobitidae ${ }^{[11-12]}$

84 花斑副沙䱊 Parabotia fasciata Dabry

85 武昌副沙鱾 Parabotia banarescui (Nalbant)

86 长薄鳅 Leptobotia elongate (Bleeker)

87 紫薄䱊 Leptobotia taeniops (Sauvage)

88 花鲉 Cobitis taenia Linnaeus

89 中华鱾 Cobitis sinensis Sauvage et Dabry

90 大斑鲀 Cobitis macrostigma Dabry

91 泥鲰 Misgurnus anguillicaudatus (Cantor)

92 大鳞副泥鲀 Paramisgurnus dabryanus Sauvage 平鳍鲉科 Homalopteridae ${ }^{[8]}$

93 犁头鱾 Lepturichthys fimbriata (Günther) 鲇科 Siluridae ${ }^{[6,9]}$

94 鲇 Silurus asotus Linnaeus

95 大口鲇 Silurus soldatovi meridionalis Chen 胡子鲇科 Clariidae

96 胡子鲇 Clarias fuscus (Lacepède)

魭科 Bagridae ${ }^{[6,9]}$

97 黄颖鱼 Pelteobagrus fulvidraco (Richardson)

98 长须黄颡鱼 Pelteobagrus eupogon (Boulenger)

99 瓦氏黄滪鱼 Pelteobagrus vachelli (Richardson)

100 光泽黄滪鱼 Pelteobagrus nitidus (Sauvage et Dabry)

101 长吻鯔 Leiocassis longirostris Günther

102 粗唇鯔 Leiocassis crassilabris Güther

103 圆尾拟鲿 Pseudobagrus tenuis (Günther)

104 乌苏里拟鲿 Pseudobagrus ussurienis (Dybowski)

105 细体拟鲿 Pseudobagrus pratii (Günther)

106 白边拟鲿 Pseudobagrus albomarginatus (Rendhal)

107 凹尾拟魭 Pseudobagrus emarginatus (Regan)

108 大鳍鳠 Mystus macropterus (Bleeker)

针头鮸科 Amplycipitidae ${ }^{[6,9]}$

109 黑尾鲔 Liobagrus nigricauda Regan

110 司氏鲔 Liobagrus styani Regan

111 鳗尾鲔 Liobagrus anguillicauda Nichols

112 白缘鲔 Liobagrus marginatus (Günther)
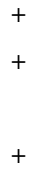

$+$

$+$

$+$

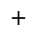

$+$

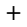

$+$

$+$
$+$ 
续表 1

鳉科 Cyprinodontidae

114 中华青鳞 Oryzias latipes sinensis (Chen, Uwa et Chu) ${ }^{[10]}$

鱵科 Hemirhamphidae

115 间下鮓 Hyporhamphus intermedius Cantor ${ }^{[10]}$

合鳃鱼科 Synbranchidae

116 黄鳝 Monopterus albus (Zuiew)

鮨科 Serranidae ${ }^{[6,10]}$

117 长身䱻 Coreosiniperca roulei ( Wu)

118 鲊 Siniperca chuatsi (Basilewsky)

119 大眼鲭 Siniperca kneri Garman

120 波纹鲭 Siniperca undulatus Fang et Chong

121 斑鳜 Siniperca scherzeri Steindachner

塘鳢科 Eleotridae

122 褐塘鳢 Eleotris fusca (Bloch et Schneider)

123 沙塘鳢 Odontobutis obscurus (Temminck et Schlegel)

124 小黄黝鱼 Micropercops swinhonis (Günther) ${ }^{[10]}$

鰕虎鱼科 Gobiidae ${ }^{[10]}$

125 粘皮鲻鰕虎鱼 Mugilogobius myxodermus (Herre)

126 子陵吻鰕虎鱼 Rhinogobius giurinus (Rutter)

127 波氏吻鰕虎鱼 Rhinogobius cliffordpopei (Nichols)

斗鱼科 Belontiidae

128 圆尾斗鱼 Macropodus chinensis (Bloch)

129 叉尾斗鱼 Macropodus opercularis (Linnaeus)

鳢科 Channidae

130 乌鳢 Channa argus (Cantor)

131 月鳢 Channa asiatica (Linnaeus)

刺䱊科 Mastacembelidae

132 中华刺鲀 Mastacembelus sinensis (Bleeker) ${ }^{[10]}$

舌鳎科 Cynoglossidae

133 窄体舌鳎 Cynoglossus gracilis Günther

134 短吻舌鳎 Cynoglossus trigrammus (Gray) ${ }^{[10]}$

鲀科 Tetraodontidae

135 弓斑多纪鲀 Takifugu ocellatus (Linnaeus) ${ }^{[10]}$

136 暗纹多纪鲀 Takifugu fasciatus (Abe $)^{[10]}$

\section{2 鱼类生态类型}

按鱼类生活史各阶段栖息的水域环境条件的差异,鄱阳湖鱼类大致可以分为以下四种生态类型: (1) 湖泊定居性鱼类, 大多数种类属这一类群, 它们的繁殖、生长、发育过程都在鄱阳湖中进行, 如鲤、鲫、红鳍原 鲌、黄滪鱼、鲇、乌鳢等,这些鱼类是鄱阳湖渔业的重要基础. (2) 江湖洄游性鱼类,它们在湖中生长发育,但 必须到江河适宜的流水中产卵繁殖，进行江湖之间的洄游活动; 青鱼、草鱼、䱠、鳙、鳡、鳤、赤眼䲡等均属这 一类型, 其中前四种是我国淡水养殖的主要对象, 在鄱阳湖渔业中有着重要意义. (3) 过河口洄游性鱼类, 鲚、鲥和鳗鳎是这次调查期间见到的海淡水洄游性鱼类,前两种具有溯河洄游习性, 它们在海水中生长、发 
育, 性成熟后必须到淡水中繁殖产卵; 后一种恰好与前者相反, 属降河洄游类型, 性成熟后必须到海水中繁 殖产卵, 幼鱼溯河到湖泊中生长、发育. (4) 溪流性鱼类, 如中华纹胸鮡、胡子鲇和月鳢等, 它们原本生活在 鄱阳湖水系上游的水质清澈的溪流中, 后随流水人湖, 经过长期适应而生存下来 ${ }^{[5]}$.

\section{3 渔具渔法}

鄱阳湖水面大,生境复杂, 既有流水也有静水,既有浅水洲滩也有深水沟潭, 能适应不同生态习性的鱼 类栖息和繁衍. 在鄱阳湖,为了捕捞不同生境的鱼类,相应的渔具渔法也非常多,初步查明有 40 余种,主要 渔具渔法有:网筙、电捕鱼、虾毫、刺网、卡子、饵钓、虾托等 (表 2).

表 2 鄱阳湖渔具渔法名录

Tab. 2 List of fishing gears used in Lake Poyang

\begin{tabular}{|c|c|c|c|}
\hline 类别 & 渔具名称 & 类别 & 渔具名称 \\
\hline \multirow[t]{3}{*}{ 刺网类 } & 浮刺网 & 钩卡类 & 饵钓 \\
\hline & 沉刺网 & & 毛钓 \\
\hline & 拖刺网 & & 卡钓 \\
\hline 围网类 & 稀围网 (大网) & & 挂钩 \\
\hline \multirow[t]{3}{*}{ 拖网类 } & 银鱼拖网 & & 拖钩 \\
\hline & 毛鱼拖网 & & 甩钩 \\
\hline & 虾拖网 & & 滚钩 \\
\hline \multirow[t]{4}{*}{ 张网类 } & 手罾 & 投刺类 & 鱼叉 \\
\hline & 桩张网 & & 灯叉 \\
\hline & 针张网 & & 镱 \\
\hline & 套张网 & & 泥鳅针叉 \\
\hline \multirow[t]{2}{*}{ 敷网类 } & 扳罾 (大罾) & 抓耙类 & 抓耙 (耙子) \\
\hline & 障网 & 窝漕类 & 把场( 打把) \\
\hline \multirow[t]{2}{*}{ 掩网类 } & 撒网 & & 迷魂阵 \\
\hline & 麻罩 & 笼豪类 & 花篮 \\
\hline \multirow[t]{7}{*}{ 操网类 } & 虾托 & & 鳜管子 \\
\hline & 虾撮 & & 鳝管子 \\
\hline & 舀子 & & 裤络 (裤形管子) \\
\hline & 夹杆子 & & 组络 (流水管子) \\
\hline & 赶嘪 & & 虾毫 (虾笼、虾篓子) \\
\hline & & 禽兽类 & 鸬鹚 \\
\hline & & 其 他 & 电捕鱼 \\
\hline
\end{tabular}

由于坚固耐用的合成纤维材料广泛使用,在 50 年代经常可见的竹箔渔法已被网䈤所取代. 目前网䈤 在鄱阳湖是最常见、数量最多的渔具. 在鄱阳湖, 根据网目大小可将网穊分为密眼和稀眼两类. 前者网目直 径在 5-10 mm, 主要在沿岸带作业. 后者网目直径一般为 $15-30 \mathrm{~mm}$, 通常安置在水较深的水域. 因受长江 水位影响,鄱阳湖水位落差大,季节性明显,5月水位上涨,9月开始下降. 因此网簖捕捞旺季在春、夏、秋鱼 类繁育和生长季节. 秋末至春初, 由于水位下降, 原先能插网篓的许多水域先后变成了无水的湖滩, 再加上 水温低,鱼类活动减弱,网䈤鱼产量往往很低.

除了网筙外, 在秋末至仲春, 电捕也是鄱阳湖常见的一种渔捞方式, 电设备通常安装在带船尾机的木 船上，俗称 “电捕船”。一条电捕船一般以一台 $9-15 \mathrm{~kW}$ 的 195 型柴油机为动力，带一台有整流装置的发 电机. 电捕船可独立操作, 亦可与围网等配合, 以便提高捕捞效果. 电捕鱼始于 20 世纪 70 年代, 进人 80 年 代后,鉴于其对鱼类资源造成较大损害, 明确列为禁止使用的有害渔具. 但随着小型柴油机和发电机的普 
及,加之该渔法简便易行且效率高,许多人仍非法使用,禁而不止.

此外, 由于湖面广阔, 流水作业生境多, 拖网、围网类渔具经常可见,各种形式的张网也常使用. 在秋冬 退水期间,各种操网也广泛应用于湖滩草洲中.

表 3 鄱阳湖稀网箱(网目 $15-20 \mathrm{~mm}$ ) 渔获物组成

Tab. 3 Fish composition of catch by net-weir with mesh size of $15-20 \mathrm{~mm}$ in Lake Poyang

\begin{tabular}{|c|c|c|c|c|}
\hline \multirow{2}{*}{ 鱼 类 } & \multicolumn{2}{|c|}{ 都昌水域 (1998 年 11 月) } & \multicolumn{2}{|c|}{ 波阳水域 (2000 年 4 月) } \\
\hline & 重量 ( kg) & 百分比 (\%) & 重量 ( kg) & 百分比 (\%) \\
\hline 鲫 & 248.5 & 32.8 & 102 & 38.5 \\
\hline 红鯺原鲌 & 129.5 & 17.1 & 41 & 15.6 \\
\hline 黄颡鱼 & 13 & 1.7 & 27 & 10.2 \\
\hline 䱗 & 159.6 & 21.1 & 18 & 6.8 \\
\hline 草鱼 & 15 & 2 & 3 & 1.2 \\
\hline 鲢 & 4 & 0.5 & 2 & 0.6 \\
\hline 鲤 & 2.5 & 0.3 & 36 & 13.5 \\
\hline 鲌类 & 14.5 & 1.9 & 10 & 3.8 \\
\hline 鲇 & 8 & 1.1 & 1 & 0.2 \\
\hline 其他 ${ }^{*}$ & 162.4 & 21.5 & 25 & 9.6 \\
\hline 合计 & 757 & 100 & 265 & 100 \\
\hline
\end{tabular}

*主要包括鱊类、沙塘鳢和蛇鮈类

\section{4 渔获物调查}

2.4.1 网穊渔获物组成根据 1997 年和 1998 年冬季、2000 年春季在湖口、都昌和波阳水域调查, 稀网䈤渔 获物中尽管有草鱼、翘嘴鲌、蒙古鲌、鲤、鲇、鲢等大中型鱼类,但它们所占的比例很小 $(<20 \%)$, 绝大部分 是小型鱼类 $(>80 \%)$, 主要包括鲫、黄颡鱼、红鯺原鲌、㛑、鳑鲏鱼类等 (表 3 ). 密网篓中几乎都是小型鱼 类,黄颡鱼、鲫、餐、蛇鮈类的比例较高 (表 4 ).

表 4 鄱阳湖密网䈤(网目约 $5 \mathrm{~mm}$ ) 渔获物组成

Tab. 4 Fish composition of catch by net-weir with mesh size of about $5 \mathrm{~mm}$ in Lake Poyang

\begin{tabular}{|c|c|c|c|c|c|c|}
\hline \multirow{2}{*}{ 鱼 类 } & \multicolumn{2}{|c|}{ 湖口水域（1997 年 12 月） } & \multicolumn{2}{|c|}{ 湖口水域（1998 年 11 月） } & \multicolumn{2}{|c|}{ 都昌水域（1998 年 11 月） } \\
\hline & 重量 ( kg) & 百分比 (\%) & 重量( kg) & 百分比 (\%) & 重量 ( kg) & 百分比 (\%) \\
\hline 鲫 & 0.5 & 2.2 & 1.8 & 12.9 & 6.5 & 14.9 \\
\hline 黄颡鱼 & 9.4 & 40.5 & 6.3 & 45 & 3.9 & 8.9 \\
\hline 红鰙原鲌 & & & & & 3.1 & 7.1 \\
\hline 蛇鮈类 & 2.8 & 12.1 & 0.4 & 2.8 & 5.2 & 11.9 \\
\hline 㛑和似鲚 & 6.3 & 27.2 & 2.8 & 19.9 & 20.2 & 46.4 \\
\hline 短领鲚 & 3.2 & 13.8 & 2.1 & 15 & 2.1 & 4.8 \\
\hline 鳜 & 0.7 & 3 & & & & \\
\hline 其他 $^{1)}$ & 0.3 & 1.3 & 0.6 & 4.3 & 2.6 & 6 \\
\hline 合计 & 23.2 & 100 & 14 & 100 & 43.6 & 100 \\
\hline
\end{tabular}

1 ）主要包括鱊类和鰕虎鱼类

2.4 .2 电捕船渔获物组成 2000 年 4 月, 对两条联营的电捕船的渔获物进行了较详细的调查, 两船上共有 5 人, 其中 1 人为雇工, 协助捞鱼. 每天的支出主要是机油、柴油、雇工及生活等费用, 每天销售的各类鱼数量 和金额均有记载. 统计了 $30 \mathrm{~d}$ (3 月 -4 月) 的收人和支出数据, 累计捕鱼 $1990 \mathrm{~kg}$, 总产值 9660 元, 总支出 2670 元, 平均每天捕鱼 $66.3 \mathrm{~kg}$, 产值 322 元, 支出 89 元,毛利润 233 元. 电捕鱼的主要对象是黄颡鱼,在渔 
获物中其产量占 $69.6 \%$,产值占 $81.0 \%$, 其次是鲫 $(14.8 \%, 8.3 \%$ ) 和小杂鱼 $(11.1 \%, 3.5 \%$ ), 再次是鲌类 $(3.1 \%, 3.6 \%)$. 鲤、鲇、乌鳢、鲧等也较常见, 但在产量和产值中所占的比例很小.

表 5 鄱阳湖刺网(网目 $5-10 \mathrm{~cm}$ ) 渔获物组成

Tab. 5 Fish composition of catch by gill nets with mesh size of $5-10 \mathrm{~cm}$ in Lake Poyang

\begin{tabular}{|c|c|c|c|c|}
\hline \multirow{2}{*}{ 鱼 类 } & \multicolumn{2}{|c|}{ 湖口水域（1998 年 11 月） } & \multicolumn{2}{|c|}{ 都昌水域（1998 年 11 月） } \\
\hline & 重量 $(\mathrm{kg})$ & 百分比 (\%) & 重量 ( kg) & 百分比 (\%) \\
\hline 草鱼 & 28 & 36.6 & 3 & 5.4 \\
\hline 团头鲂 & 1.2 & 1.6 & & \\
\hline 鲢、鳙 & 25 & 32.6 & 14 & 25.3 \\
\hline 鲤 & 7 & 9.1 & 23 & 41.5 \\
\hline 鮥 & 5.6 & 7.3 & 4 & 7.2 \\
\hline 鲌类 * & 8 & 10.4 & 11.4 & 20.6 \\
\hline 其他 ${ }^{* *}$ & 1.8 & 2.3 & & \\
\hline 合计 & 76.6 & 100 & 55.4 & 100 \\
\hline
\end{tabular}

*包括翘嘴鲌、蒙古鲌和达氏鲌; **包括花䱻、黑尾䱀、赤眼鳟和鳊.

2.4.3 刺网渔获物组成 刺网目大一般为 5-10 cm, 多在冬、春季使用. 根据 1998 年在湖口和都昌水域的 调查,刺网渔获物中草鱼、鲢、鳙、鲤和鲌类所占的比例较高 (表 5 ), 鳡也是重要的渔获对象. 这里需要特别 说明的是,草鱼、鲢和鳙的较高比例可能与 1998 年长江中下游流域的特大洪水有关, 因为据渔民反映,这次 洪水使鄱阳湖周边的许多池塘、湖汊、网拦被淹, 人工养殖的鲢、鳙和草鱼大量逃逸.

2.4 .4 钧具渔获物组成饵钓一般以虾类、小鱼或鸭毛为诱饵, 是专一性较强的渔具, 主要捕捞肉食性鱼 类. 根据 2000 年 4 月在鄱阳湖都昌水域调查, 饵钓 (以鸭毛为诱饵) 渔获物中翅嘴鲌占 $57.5 \%$, 达氏鲌 $17.5 \%$,鳡 $15.0 \%$ (表 6 ).

表 6 鄱阳湖都昌水域钧具渔获物组成

Tab. 6 Fish composition of catch by fishhook in Lake Poyang

\begin{tabular}{|c|c|c|c|c|c|c|}
\hline 种类 & 鳡 & 攰嘴鲌 & 蒙古鲌 & 达氏鲌 & 其他 ${ }^{*}$ & 合计 \\
\hline 重 量 (kg) & 6 & 23 & 2.5 & 7 & 1.5 & 40 \\
\hline 百分比 (\%) & 15.0 & 57.5 & 6.3 & 17.5 & 3.8 & 100 \\
\hline
\end{tabular}

*包括红鯺原鲌、鲇、餐和似鲚.

\section{5 渔产量估算及动态}

图 1 显示了自 1950 年以来的各个时期的年均渔产量, 在 60 年代以前, 年均渔产量大约是 $88 \mathrm{~kg} / \mathrm{hm}{ }^{2}$, 在 70 年代, 渔产量明显下降,约为 $62 \mathrm{~kg} / \mathrm{hm}^{2}$, 随后逐步上升, 至 90 年代, 高达 $198 \mathrm{~kg} / \mathrm{hm}^{2}$. 如果翻阳湖面积 的统计不包括其周围现已分隔开来的众多湖汊,那么 80 年代以后的渔产量估算可能有所偏高, 因为各县市 统计的天然捕捞量可能包括了境内其他湖泊天然捕捞量.

\section{3 讨论}

\section{1 鱼类组成的变化}

在本次调查期间记录的鱼类 101 种,其中新记录的鱼类有 6 种 (表 1 ), 全部是小型鱼类. 在新记录的鱼 类中,除洞庭小鳔鮈和短须鱊稀少外,其它 4 种很常见,数量较多. 这些鱼类在以往的多调查中没有采到, 可能与采样地点、采样渔具和采样强度有关.

与以前的调查相比,总计 35 种鱼类在本次调查期间没有采到, 它们是: 中华鲟、白鲟、窄体舌鳎、短吻舌 鳎、弓斑多纪鲀和暗纹多纪鲀 6 种洄游性和半洄游性鱼类, 尖头鲴、唇鱼骨、长麦穗鱼、短须领须鮈、北方铜 
鱼、圆筒吻鮈、宜昌鱾鮀、台湾光唇鱼、光唇鱼、稀有白甲 鱼、寡鳞鱊、巨口鱊、长身鱊和革条副鮦 14 种鲤科鱼类,

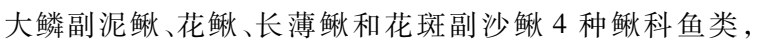
粗唇鮸、乌苏里拟魭、白边拟魭、凹尾拟魭、鳗尾鲔、白缘 鲔 6 种魭科鱼类, 乔氏新银鱼、胭脂鱼、犁头鲀、褐塘鳢 和叉尾斗鱼 5 种鱼类 (表 1 ). 这些鱼类没有采到, 并不 一定意味着它们在鄱阳湖已经消失, 可能是因为种群处 于濒危状态, 或数量特别少, 或分布范围狭窄, 生境特殊, 以致在渔具种类和数量、采样范围和强度有限的情况下 一时难以捕到.

\section{2 渔业利用问题}

鄱阳湖是一个过水性吞吐型湖泊, 水位落差大, 跨

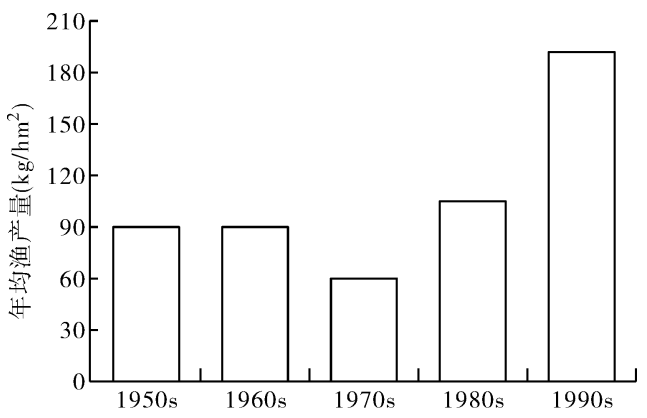

图 1 自从 20 世纪 50 年代以来鄱阳湖渔产量动态

Fig. 1 Changes in annual fish catch from

Lake Poyang since 1950s 界矛盾多, 至今没有开展人工放流, 因此渔业是以鱼类 的自然种群为基础, 鱼的食料不是依靠投饵而是仰给于天然的. 对于这种大型通江湖泊,探讨鱼类资源的 合理利用问题具有重要的意义. 根据鄱阳湖渔业资源的特征和利用现状,提出以下几点建议:

(1) 高度重视凶猛性鱼类资源的保护与增殖, 以期利用丰富的小型鱼类和虾类资源, 提高鱼产品质量 和经济效益.

从生活史对策看, 鄱阳湖鱼类可分为两大类群:一类是小型鱼类,主要特点是个体小,性成熟早,相对繁 殖力较高, 寿命较短, 大都属于 $r$-对策者; 另一类是大中型鱼类, 主要特点是个体大, 性成熟较晚, 相对繁殖 力较低,寿命较长,一般属于 $k$-对策者. 如果将性成熟年龄为 1 年, 且首次性成熟时平均体重小于 $50 \mathrm{~g}$ 的 鱼类视为小型鱼类,其余的视为大中型鱼类,那么鄱阳湖小型鱼类种数约占鱼类总种数的 $60 \%$ 以上. 在小 型鱼类中, 具有较高的经济和渔业价值的只有鲫、红魦原鲌、黄滪鱼、银鱼和鲚类, 其它的大多数小型鱼类因 价格低没有渔业意义. 在大中型鱼类中, 凶猛鱼类 (以小鱼和虾为主要食物的鱼类的通称) 约占一半. 目 前, 在鄱阳湖中, 具有一定产量且价值高的凶猛性鱼类有鳡、趐嘴鲌、蒙古鲌、达氏鲌、红鯺原鲌、黄颞鱼、鲇、 鲜和乌鳢等,但在每年总产量中所占的比例非常低. 这些鱼类在空间或营养生态位上存在一定的分化,能 利用不同水层和生境中低值的小型鱼类和虾类资源. 因此,在渔业资源管理中应该引起高度重视,通过加 强繁殖保护措施, 不断恢复和增强凶猛性鱼类种群, 将低值、丰富的小型鱼类和虾类资源转化为名贵高档的 水产品.

(2) 积极开展鄱阳湖鱼类人工放流, 增殖日益衰退的 “四大家鱼”资源, 保障通江湖泊渔业的可持续 发展.

在上个世纪 60 年代以前,青鱼、草鱼、鲢和鳙“四大家鱼” 是鄱阳湖渔业的重要基础. 随着三峡大坝建 成后, 长江重庆以下产卵场消失, 重庆以上产卵场规模虽有增大, 但鱼苗被拦截在库区; 长江中游产卵场可 能因三峡建坝后洪峰低平和冷水下泄而减少, 产卵期推迟, 繁殖期缩短, 鱼苗补充量减少 ${ }^{[13]}$. 在鄱阳湖生 长肥育的亲鱼性成熟后, 可在赣江中上游产卵, 但万安电站的修建严重影响赣江产卵场和产卵规模 ${ }^{[5]}$. 因 此, 鄱阳湖通过长江和赣江得到的“四大家鱼”补充量会越来越少; 事实上, “四大家鱼”种群已处于严重衰 退之中 ${ }^{[5]}$.

鱼类放养是自然水体增殖以及保护和维持自然种群数量的主要手段. 在今后鄱阳湖渔业管理中,应有 计划、有步骤地开展 “四大家鱼” 人工放流, 以期恢复和增殖资源, 同时合理利用湖泊中丰富的高等水生植 物、浮游生物和底栖动物, 保障通江湖泊渔业的可持续发展.

(3) 加强对禁渔区和禁渔期的管理,严格控制捕捞强度和捕捞规格,切实做好重要土著鱼类资源的繁 殖保护.

鄱阳湖鲤、鲫和银鱼的产卵场基本摸清 ${ }^{[5]}$, 今后需要重点调查尧嘴鲌、蒙古鲌、达氏鲌、红鯺原鲌、黄滪 鱼、鲇、鳜和乌鳢等重要名贵鱼类的产卵场分布、产卵高峰期和产卵持续时间,研究它们的渔业生物学特性, 跟踪监测鱼类资源的动态变化. 在这些研究的基础上,确定禁渔区和禁渔期,制定捕捞规格,控制捕捞强 
度; 通过这些措施,切实加强重要土著鱼类资源的繁殖保护和增殖, 保证鱼类资源的可持续利用和渔业的可 持续发展.

致谢: 在野外调查中得到了江西省渔政管理局领导和职工, 特别是吴鼎盛先生的帮助; 在某些鱼类鉴定、中 文名和学名订正中, 承蒙本所乐佩奇研究员和曹文宣院士的指教,在此一并表示诚挚的感谢!

\section{4 参考文献}

[1] 郭治之. 波阳湖鱼类调查报告. 江西大学学报 (自然科学版), 1964,(2): $121-130$.

[2] 郭治之, 刘瑞兰. 江西鱼类研究. 南昌大学学报 (理科版). 1995,19 (4) : $222-232$.

[3] 湖北省水生生物研究所. 长江鱼类. 北京:科学出版社, 1976: 1-243.

[4] 《鄱阳湖研究》编委会. 鄱阳湖研究. 上海: 科学技术出版社, 1988: $553-554$.

[5] 朱海虹,张 本. 鄱阳湖. 合肥: 中国科学技术大学出版社, 1997: 146-169.

[6] 朱松泉. 中国淡水鱼类检索. 南京: 江苏科学出版社,1995: $1-549$.

[7] 陈宜瑜等. 中国动物志一一硬骨鱼纲. 鲤形目 (中). 北京: 科学出版社, 1998: 1-531.

[8] 乐佩奇等. 中国动物志一一硬骨鱼纲. 鲤形目 (下). 北京: 科学出版社, 2000: $1-661$.

[9] 褚新洛,郑葆珊,戴定远等. 中国动物志 - 硬骨鱼纲 鲇形目. 北京: 科学出版社, 1999: 1-230.

[10］伍汉霖,邵广昭,赖春福. 拉汉世界鱼类名典. 台湾: 水产出版社, 1999: $1-1028$.

[11] 陈景星. 中国沙鱾亚科鱼类系统分类的研究. 动物学研究, 1980,1(1):3-26.

[12] 陈景星. 中国花鳅亚科鱼类系统分类的研究. 见: 中国鱼类学会编. 鱼类学论文集 (第一辑), 北京: 科学出版社, $1981: 21-32$.

[13] 曹文宣, 余志堂. 三峡工程对长江鱼类资源影响的初步评价及资源增殖途径的研究. 见: 长江三峡工 程对生态与环境影响及其对策研究论文集. 北京: 科学出版社,1987:3-17. 